\title{
Optimization of a Method for Imaging Drosophila Embryos with a Variable Pressure Scanning Electron Microscope
}

\author{
Michelle Gignac*, Xiu Chen ${ }^{* *}$, Stephen Lockett*, Kunio Nagashima* \\ * National Cancer Institute-Frederick, SAIC-Frederick, Image Analysis Laboratory, Frederick MD \\ ** National Cancer Institute-Frederick, Laboratory of Immunobiology, Frederick MD
}

Drosophila have long been used as a model for basic genetic and protein research. Both the embryo and adult flies are used in these studies. The morphology of the embryo is of specific interest to the researchers, since modification of the genome can lead to dramatic changes in the development of the embryo. We have imaged changes in the morphology of the embryos using the scanning electron microscope (SEM).

To image the Drosophila embryos the outer vitelline membrane must first be removed. This can be done through either a hand peeling or a methanol "popping" method [1]. The samples were processed in the traditional technique; dehydrated through graded ethanol washes, dried, placed on SEM stubs and coated with $\mathrm{Pd} / \mathrm{Au}$ [2]. When we processed the embryos with this method, many artifacts were observed (Figure 1). We felt that the variable pressure SEM (VP-SEM) would be a more appropriate means to image the samples and obtain artifact free images.

The first set of samples for VP-SEM imaging were prepared by the hand peeling method. These samples were imaged on the Hitachi S-3000N VP-SEM at 80 to $90 \mathrm{~Pa}$. Some of the embryos were observed collapsed (Figure 2). The collapse of the samples was found to be independent of pressure and time (data not shown). We concluded that the hand rolling method could damage the internal structures of the embryos and that minimal pressure is needed for the collapse of the samples. The collapse may occur in the initial pumping down of the instrument.

Samples were then submitted using the methanol "popping" method. Better results were observed, but there were still some artifacts. We then began to fix the samples with $2 \%$ gultaraldehyde for at least $4 \mathrm{hrs}$ before the removal of the membrane [3]. This eliminated most of the artifacts that we had previously seen (Figure 3). We concluded that pre-fixation results in better sample quality.

The VP-SEM is a superb tool for imaging hard to process samples. It eliminates dehydration artifacts and allows one to image samples in a more natural setting. But, the VP-SEM may bring different artifacts and challenges to viewing samples. Methods need to be optimized to obtain the highest quality results.

\section{References}

[1] M. Ashburner, Drosophila A Laboratory Manual, Cold Spring harbor Laboratory Press, Cold Spring Harbor, NY, 1989.

[2] J.J. Bozzola, L.D. Russell, Electron Microscopy Principles and Techniques for Biologists, $2^{\text {nd }}$ ed., Jones and Bartlett Publishers, Sudbury, MA, 1999.

[3] K. Barrett, M. Leptin, J. Settleman, Cell. 91 (1997) 905. 

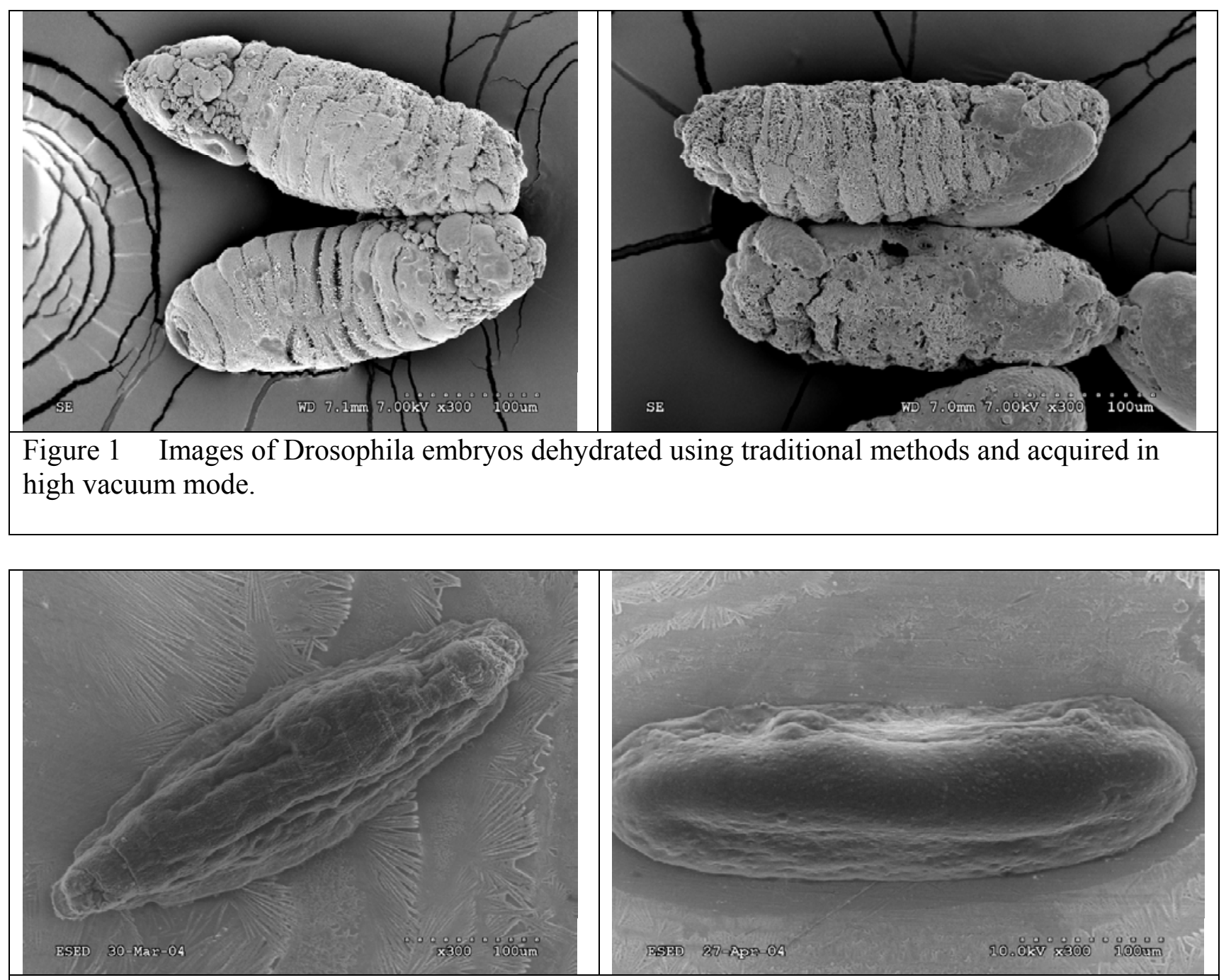

Figure 2 Images of Drosophila embryos taken in the VP mode and processed by the hand peeling method.

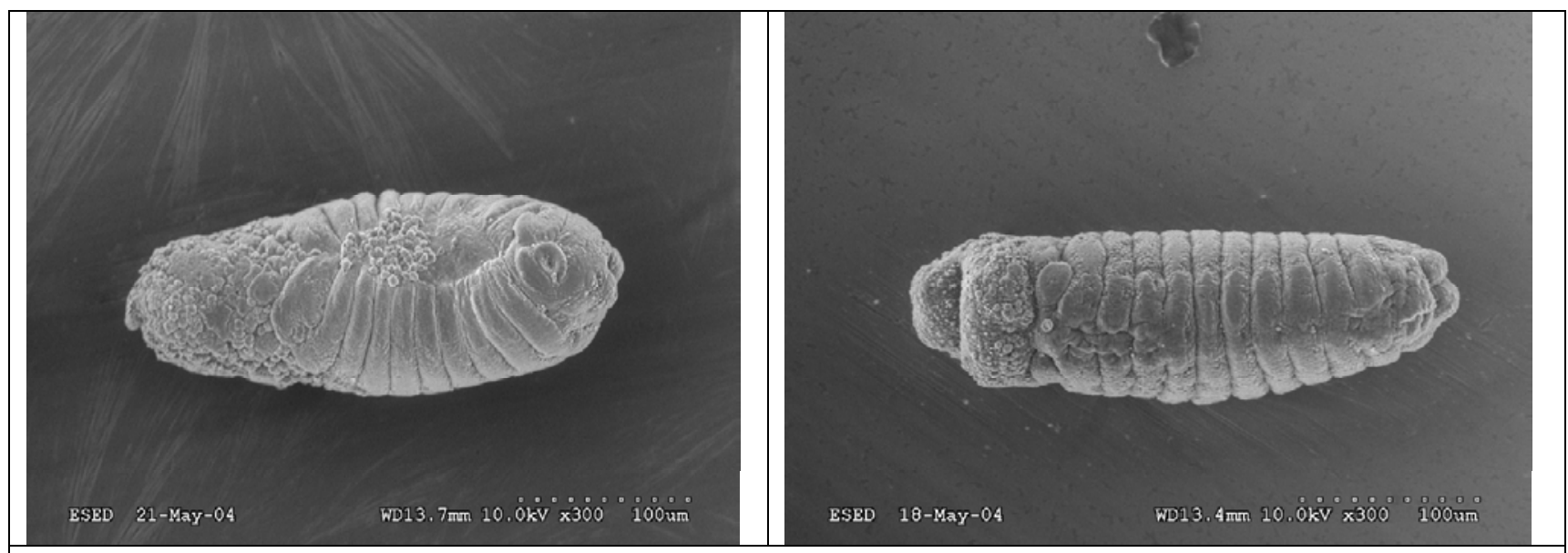

Figure 3 Images of Drosophila embryos taken in the VP mode and processed with the methanol popping method.

All images were taken on the Hitachi S-3000N SEM. 